\title{
I Consensus for the Management and Treatment of Hepatitis B Carried Out by the Brazilian Society of Infectious Diseases
}

\author{
Coordination \\ Evaldo Stanislau Affonso de Araújo \\ Organization \\ Brazilian Society of Infectious Diseases - Hepatitis Committee \\ Antonio Alci Barone, Evaldo Stanislau Affonso de Araújo, Fernando Lopes G. Junior, \\ João Silva Mendonça Marcelo Simão Ferreira and Roberto Focaccia
}

Aline G. Vigani

Ana Teresa Rodrigues Viso

André Cosme de Oliveira

Antonio Alci Barone

Edgard DeBortholi

Edson Abdala

Evaldo Stanislau Affonso de Araújo

Evandro S. de Melo

Fátima Mitiko Tengan

Fernando Lopes Gonçales Jr

João da Silva Mendonça

José Carlos Fonseca

This paper reports on the conclusions reached by the Hepatitis Committee of the Brazilian Society of Infectious Diseases in their I Consensus for the Management and Treatment of Hepatitis B. The subjects considered most relevant or controversial among those discussed by the Consensus Group*, which met in the city of São Roque on July 21-23, 2006, are summarized in this report. A systematic review on topics related to hepatitis $B$ was carried out and published in the Brazilian Journal of Infectious Diseases. We strongly recommend that readers consult the Proceedings of the Consensus Meeting in which a full, detailed report on the topics discussed is published, whereas in the Consensus, these topics are concisely and objectively summarized.

\section{Definition of Chronic Hepatitis B}

According to the definition of the Brazilian Society of Infectious Diseases (Sociedade Brasileira de Infectologia - SBI), chronic hepatitis B is characterized by the presence of HBsAg for more than 6 months, HBV DNA > 20,000 IU/ $\mathrm{mL}$, persistently or intermittently elevated levels of ALT, and a liver biopsy revealing portal 2 or periportal 1 necroinflammatory activity, according to the Brazilian Society of Pathology classification (or ISHAK 4 or METAVIR A1). It should be emphasized that in chronic hepatitis $B$ the intensity of the inflammatory process is dynamic and that in patients coinfected with HIV inflammatory activity may be less intense.

\section{Positivity to Anti-HBc Alone and Occult HBV Infection}

When a patient is positive for either anti-HBc IgG or total anti-HBc alone, repetition of the marker is recommended with the addition of HBsAg, all with expressed titers, and vaccination (as a booster) followed by anti-HBs quantification seven days after administration of the vaccine.

If possible, the investigation of HBV DNA is always recommendable, particularly in the following situations: in the case of coinfection with HIV, coinfection with HCV, in patients who have received multiple blood transfusions, prior to organ transplant, in more vulnerable populations, in the case of individuals who are to receive chemotherapy and in immunodepressed individuals in general.

\section{Liver Biopsy: Its Value, Indications and Use of Tissue Markers}

The objective of the liver biopsy is to: 1 ) reach a diagnosis; 2) grade and stage the disease; and 3) diagnose other diseases of the liver, particularly alcoholic or non-alcoholic steatohepatitis. Whenever possible, biopsy should be performed at the beginning of follow-up (baseline), particularly in HBeAg-negative patients with elevated ALT and in patients coinfected with HIV. Biopsy should only be carried out in HBeAg-positive patients with elevated ALT when there is doubt with respect to diagnosis or when cirrhosis is suspected but has not been confirmed by other non-invasive methods (e.g. ultrasonography).

* Reviewers who did not participate in the consensus meeting. 
Tissue marker evaluation is always recommended. The presence of HBsAg reaches sensitivity of $100 \%$. Cytoplasmic HBsAg is indicative of infection while membranous HBsAg expression indicates replication. The core antigen reaches sensitivity of $70 \%$. When nuclear, it is indicative of replication and when cytoplasmic, of high necroinflammatory activity. The $\mathrm{X}$ antigen is currently undergoing validation and appears to be correlated with a risk of evolution to hepatocellular carcinoma.

\section{Quantification of HBV DNA: Indications and Interpretation}

In view of the technical difficulties and lack of standardization of internal controls, which may result in underestimated or false-negative results, it is recommended that the same methodology should always be used for the follow-up of a patient and that the use of in-house methods should not be implemented on a wide scale. This subject is reviewed in the Proceedings published in the BJID; however, the minimum cut-off levels for the recommendation of HBV therapy are shown in Table 1 below:

\begin{tabular}{ll}
\hline $\begin{array}{l}\text { Clinical/ } \\
\text { serological status }\end{array}$ & $\begin{array}{l}\text { Minimum limit } \\
\text { for therapy }\end{array}$ \\
\hline $\begin{array}{l}\text { HBeAg-positive } \\
\text { HBeAg-negative }\end{array}$ & $\geq 20,000 \mathrm{UI} / \mathrm{mL}^{*}$ \\
$\begin{array}{l}\text { Cirrhotic patient } \\
\text { in a stable condition }\end{array}$ & $\geq 2,000 \mathrm{UI} / \mathrm{mL}^{*}$ \\
$\begin{array}{l}\text { Cirrhotic patient } \\
\text { on transplant list }\end{array}$ & Any amount/detection \\
Coinfection with HIV & $\geq 2,000 \mathrm{UI} / \mathrm{mL}^{*}$ \\
\hline
\end{tabular}

*IU is the equivalent of 5.6 copies $/ \mathrm{mL}$.

As suggested by the SBI panel, HBV DNA should be measured at the beginning of follow-up (baseline) and, in the case of patients in use of antiviral medication, every six months until non-detectability, and annually thereafter. If ALT/AST levels increase, viral load evaluation should be carried out earlier. When drugs with a high potential for the development of genotypical resistance are used, such as lamivudine, for example, more frequent evaluations of viral load may be required, principally after 12 months of continuous use.

\section{Screening for Hepatocellular Carcinoma (HCC) and Indications for Liver Transplant}

In general, patients who have acquired HBV by vertical transmission and cirrhotic patients are at greatest risk of HCC. Therefore, ultrasonography of the liver and alpha-fetoprotein measurement (which may be normal even if HCC is present) should be performed every six months. Alpha-fetoprotein $>400$ $\mathrm{ng} / \mathrm{mL}$ when no nodules are present is generally indicative of HCC. If a nodule is present and alpha-fetoprotein is $>200 \mathrm{ng} /$ $\mathrm{mL}$, diagnosis may also be confirmed. Nodules $>2.0 \mathrm{~cm}$ have the worst prognosis and may already have metastasized. Therefore, diagnosis should ideally be made when nodules are $<1.5 \mathrm{~cm}$ in diameter and, if a nodule is present, the patient should immediately be referred to a specialist center. It should be emphasized that routine biopsy is not indicated for the diagnosis of liver nodules.

Liver transplant is indicated in the case of CHILD B and C patients (or CHILD A with complications that are difficult to control), in cases of fulminant hepatitis and whenever HCC is present.

\section{Treatment of Viral Hepatitis B}

The objectives of HBV therapy are: to achieve nondetectability of HBV DNA and to turn HBsAg negative. This is usually rare and may be substituted for HBeAg-negative, or, even better, seroconversion to anti-HBe-positive.

In general, therapy is indicated for patients with high ALT in addition to the cut-off levels of HBV DNA shown above. Elevated ALT levels are usually predictive of a good response to therapy. Patients with normal ALT and detectable HBV DNA should be considered for therapy in accordance with the results of their liver biopsy, which should indicate fibrosis stage $\geq 2$ or significant necroinflammatory activity. Finally, the type of exposure of the patient should be evaluated. Those patients who were infected by vertical transmission may remain in the immunotolerance phase for decades and may seroconvert after 30 to 40 years. Such cases demand caution with respect to the indication of therapy since they may resolve spontaneously. On the other hand, in the case of patients infected by horizontal transmission in early infancy, seroconversion may occur by the end of childhood or in early adolescence. In those infected in adulthood, there is a tendency towards a better response to therapy as a result of a stronger immune response.

This analysis took the five drugs used for the treatment of chronic hepatitis B into consideration: conventional or pegylated interferon-alpha, lamivudine, adefovir and entecavir administered according to the usual therapeutic doses and regimens.

\section{Treatment of Patients Monoinfected with HBV}

Treatment-Naïve Patients

Since decisions with respect to therapeutic management depend on the presence of $\mathrm{HBeAg}$, patients have been separated into HbeAg-positive or negative subgroups, as follows:

\section{HBeAg-Positive Patients}

In this subgroup, patients are divided into those with a favorable profile for therapy and those in whom therapy is considered to be more difficult. Patients with a favorable profile are younger, have a lower viral load, no comorbidities and no cirrhosis. Those in whom treatment is considered more difficult are older, have a higher viral load, comorbidities and cirrhosis. Further details of these classifications may be found in the Proceedings of the Consensus.

In carriers of HBeAg-positive infection with a favorable profile, therapy with conventional or pegylated interferon is recommended. For those patients with a poor profile, an antiviral is used, adefovir or entecavir being those recommended. 


\section{HBeAg-Negative Patients}

HBeAg-negative patients should be treated with pegylated interferon or with the antivirals adefovir or entecavir. In the case of antivirals, the use of entecavir is recommended as the first option of therapy when the patient is treatment-naïve in view of the more favorable long-term resistance profile of this drug.

The recommendations for treatment-naïve patients are summarized in Figure 1.

\section{Patients Who Have Undergone Previous Therapy for HBV}

In patients who have already undergone previous treatment with interferon, subsequent therapy should be carried out with an antiviral, either adefovir or entecavir, taking the above-mentioned factors into consideration.

For patients treated with lamivudine who show signs of resistance (increased levels of transaminases, an increase $>1$ $\log _{10}$ in relation to the nadir of the viral load), adefovir should be added to therapy for a minimum period of 3-6 months, maintaining monotherapy with adefovir after this period. If entecavir is preferred, lamivudine should be substituted for the new drug; however, the subsequent risk of developing resistance to entecavir should be emphasized.

In patients who develop resistance to adefovir, the addition of lamivudine or its substitution for entecavir is recommended as long as lamivudine has not previously been used.

It should be emphasized that only drugs currently being used for hepatitis B therapy have been considered in this report. In the near future, novel therapeutic options (clevudine, tenofovir and emtricitabine, for example) will bring new alternatives that may result in changes to the above-mentioned sequence.

Therapeutic management is summarized in Figure 2.

\section{Cirrhotic patients}

Cirrhotic patients are routinely divided into those in whom the condition has been stabilized and those in whom it has not, i.e. patients on the transplant list. For the treatment of this group of patients, the use of conventional or pegylated interferon alpha is not recommended under any circumstances.

In the case of treatment-naïve, cirrhotic patients whose condition is stable, the use of adefovir or entecavir is recommended, and the potential of the use of this association in the future should be emphasized. In the case of patients who are resistant to lamivudine, the previously-mentioned recommendations should be followed.

In the case of treatment-naïve, cirrhotic patients whose condition is unstable, monotherapy with lamivudine, adefovir or entecavir is recommended, depending on the availability of these drugs. There is also a tendency to use a combination of drugs in the future. In the case of cirrhotic patients whose condition is unstable and who are already resistant to lamivudine, the recommendation is to add adefovir until liver transplant can be performed.

\section{HBV-HIV Coinfection}

Inclusion Criteria for Therapy

Inclusion criteria are similar to those for monoinfected patients. Liver biopsy is fundamental for the management of these cases, and should be performed, whenever possible, in all patients. Indication for the treatment of hepatitis B in this group of patients may be modified in accordance with the composition of the HAART regimen indicated for the treatment of HIV infection. Table 2 lists the basic recommendations for therapeutic management. It should be noted that adefovir has the theoretical potential to induce resistance to tenofovir through a recently described mutation, $\mathrm{K} 70 \mathrm{E}$, and that pegylated interferon is the only interferon indicated in these cases.

Figure 1. Recommendations for treatment-naïve patients.

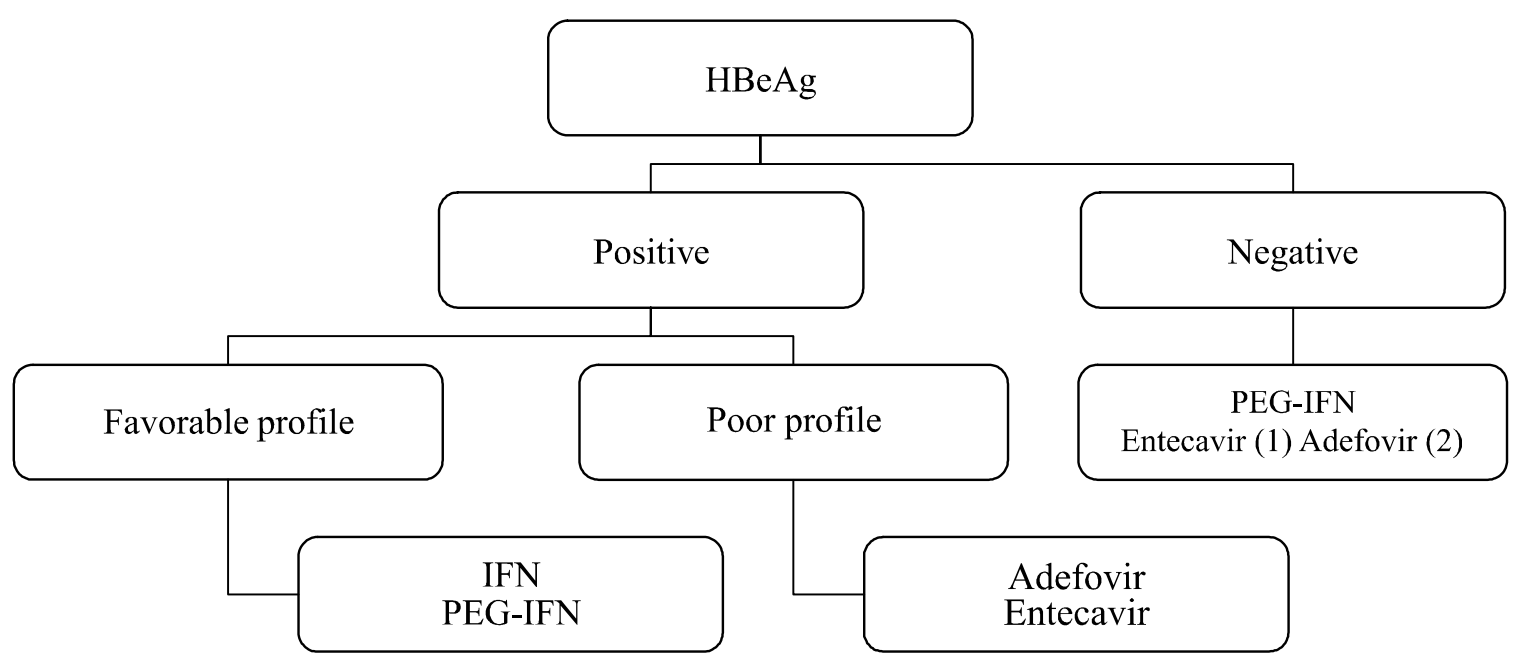


Figure 2. Therapeutic management
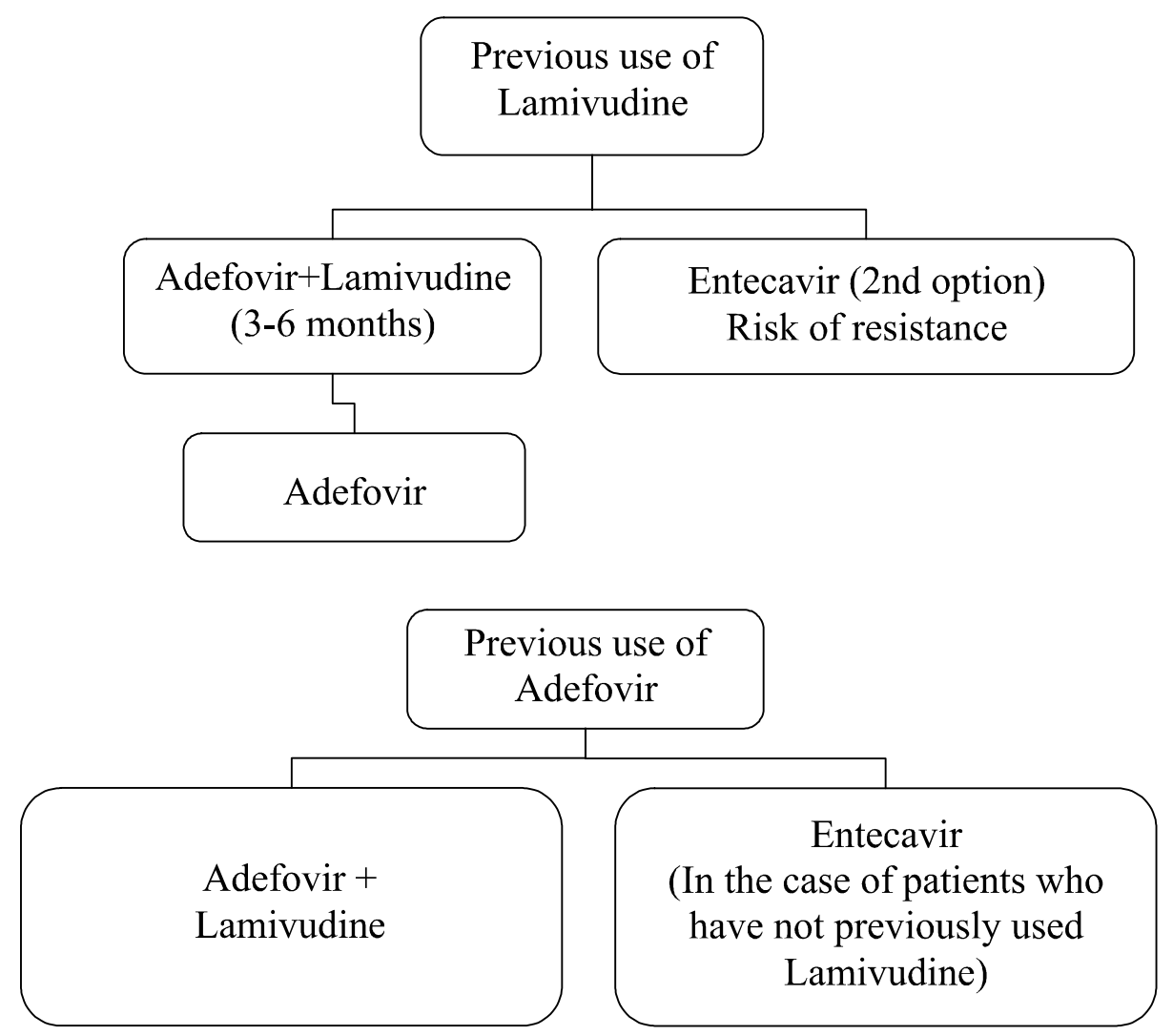

Table 2. Treatment of chronic hepatitis B in patients with HIV/HBV coinfection (Zoulim, 2006)

\begin{tabular}{|c|c|c|c|}
\hline Indication for antiviral therapy & HBeAg/PCR & Preferred Regimen & Avoid \\
\hline \multirow{5}{*}{$\begin{array}{l}\text { HBV alone (wild strain/ } \\
\text { precore mutant) } \\
\text { HIV alone }\end{array}$} & a) HBeAg-positive & IFN(PEG), ADF*, ETV & LAM, FTC, TDF \\
\hline & b) HBeAg-negative & $\mathrm{ADF}^{*}, \mathrm{ETV}$ & LAM, FTC, TDF \\
\hline & HBeAg-positive/negative + & & \\
\hline & a) HBV DNA $<10^{4}$ copies $/ \mathrm{mL}$ & Monitor & - \\
\hline & $\begin{array}{l}\text { b) } \mathrm{HBV} \text { DNA }>10^{4 \text { or } 5} \\
\text { copies } / \mathrm{mL}\end{array}$ & $\begin{array}{l}\text { HAART with TDF + } \\
\text { LAM or FTC* }\end{array}$ & $\begin{array}{l}\text { Monotherapy with } \\
\text { LAM or FTC }\end{array}$ \\
\hline $\begin{array}{l}\text { HIV + HBV (wild strain/ } \\
\text { precore mutant) }\end{array}$ & HBeAg-positive or negative & $\begin{array}{l}\text { HAART with TDF + } \\
\text { LAM or FTC }\end{array}$ & $\begin{array}{l}\text { Monotherapy with } \\
\text { LAM or FTC }\end{array}$ \\
\hline LAM-resistant HBV (YMDD) & HBeAg-positive or negative & HAART with TDF & Discontinue LAM \\
\hline
\end{tabular}

* To prevent flares due to restored immune system, particularly in cirrhotic patients. 